\title{
Computational design of prefabricated modular homes
}

\author{
Robert Doe \\ Faculty of Architecture, Planning and Design \\ The University of Sydney \\ Sydney, Australia \\ r.doe@sydney.edu.au
}

\author{
A/Prof Mathew Aitchison \\ Faculty of Architecture, Planning and Design \\ The University of Sydney \\ Sydney, Australia \\ m.aitchison@sydney.edu.au
}

\begin{abstract}
This exploratory paper takes the design solution of a prefabricated modular home series as a starting point to examine computational design tools appropriate for further improvement of the designs. Firstly, drawing on Lawson's [1] definition, the design process is defined so that design problems may be better understood. Secondly, computational design tools are assessed for their suitability to address the design problems and offer better design solutions. Computational design tools are defined for further research.
\end{abstract}

Keywords-component; computational design; prefabricated modular homes; multi-criteria optimisation; parametric modelling; simulation

\section{INTRODUCTION}

This exploratory paper takes the design solution of a modular home series as a starting point to examine computational design tools appropriate for making further improvements. This involves working to attain an integrated design solution for a wide range of criteria, an achievement defined by Lawson [1] as the mark of a good designer. The pattern of the design problem is made up of all the interactions between these criteria which further constrain what the designer may do. In understanding this pattern room for improvements may be identified.

This paper also argues that if architects could collaborate more effectively in inter-disciplinary design teams by better understanding and utilisation of these computational design tools this could improve the design process and buildings' performance by contributing towards fully integrated design solutions.

The modular home series forms a real project for a real customer, however partners and customer's details have been kept confidential (Figures 1 \& 2). The modular homes are produced by a developer for customers wanting the benefit of an architecturally designed home without the expense that custom design incurs. The client and the architect are therefore key generators of ideas and constraints which have defined the design problem and the design solution. These inputs are investigated so that the design process for this project may be understood more clearly. For example, the way these generators of design influence the design solution and the impact of internal, external and other constraints on the formation of the design solution will be examined.

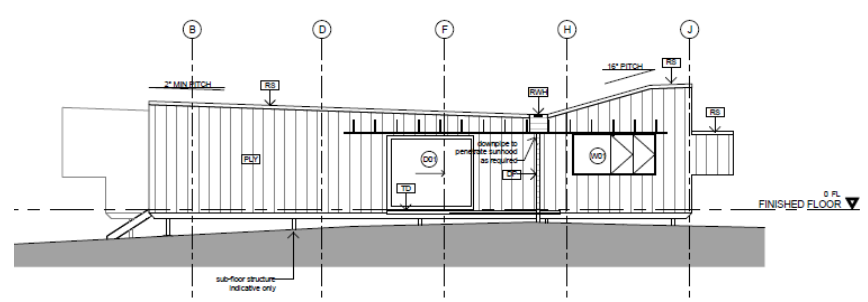

Figure 1 - modular home - side elevation

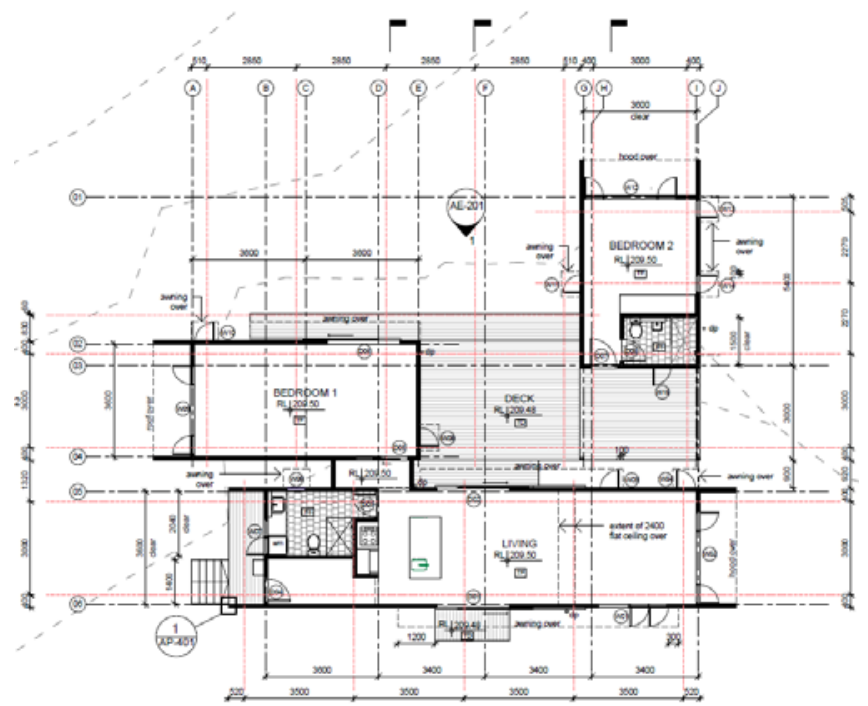

Figure 2 - modular home - plan configuration

Owing to its relevance to the design process another theme explored in this paper is the difference in thinking between the scientific realm concerned with analysis and the engineering 
and design realms mostly concerned with synthesis, even if many engineering fields are often focused on the scientific rather than the design realm. These differences influence the process and outcomes during an inter-disciplinary search for integrated design solutions and form an important context for consideration.

"The natural sciences are concerned with how things are... Design, on the other hand, is concerned with how things ought to be, with devising artefacts to attain goals. We might question whether the forms of reasoning that are appropriate to natural science are suitable also for design.” [2, Ch.3, p.59]

Simon's 'common ground' [2] is where inter-disciplinary discussions primarily occur, the space of computational interaction. Therefore, because an examination is made of computational design tools appropriate to the design process and the design solutions investigated, the tendency of these tools to favour or hinder these different ways of thinking is addressed. However, the benefits of using computational design tools and defining those which are most appropriate to this particular design problem are the two main foci of this paper. McGill [3] defines these benefits as follows:

- Generation of multiple design ideas or solutions.

- Provision of a greater depth of understanding of a design.

- Enabling the designer to make more informed decisions when developing and revising a design, and during the entire design process.

Nevertheless, the risks in believing that computational design, in its present form, may be a replacement for the skills of the human designer are not ignored. As Christopher Alexander [4] has pointed out:

"The only medium which is truly fluid, which allows the design to grow and change as new patterns enter it, is the mind.” [4, Ch.21, p.422]

\section{DESIGN PROCESS AND DESIGN PROBLEM}

The design problem and design process are deeply interlinked. A better understanding of this interconnection should begin to reveal how and by what computational design methods the modular home series can be improved. The process of design of the modular homes is as follows, with reference to Lawson's [1] 'generators' of design which influence design ‘constraints’ (Figure 3).

The client, a private developer, is especially keen on 'designer' generation of ideas as a point of difference for this particular product, namely: affordable architect-designed homes. The emphasis is on the 'client' as a generator of design input over that of the 'user' although there is some limited customisation involved with finishes and fittings. The 'legislator' generator influences are similar to those on other residential projects. These generators contribute to the constraints which define design problems and they may be 'internal', 'external', 'radical', 'practical', 'formal' or 'symbolic' in nature.

Although we might disagree with the model defined by Lawson [1] we have applied this as proposed in order to test its usefulness. The 'radical' constraints are those dealing with the primary purpose of the object being designed, the relationship and arrangement of spaces to make a home. 'Formal' constraints include rules about proportion, form, colour and texture and are the result of client and designer interaction, with limited user input as noted above. 'Practical' constraints deal with the reality of producing, making or building the modular homes, which is largely dealt with by the builder in this case, but it also includes the technical performance of the modular homes which are defined by the designers and engineers. 'Symbolic' constraints, perhaps distinguished by style, are chosen be the architect who envisions and designs the series. For residential projects like this experienced designers, familiar with 'internal' constraints such as varied types of internal arrangement, focus on the 'external' constraints - the site's physical characteristics, its context, orientation and location.

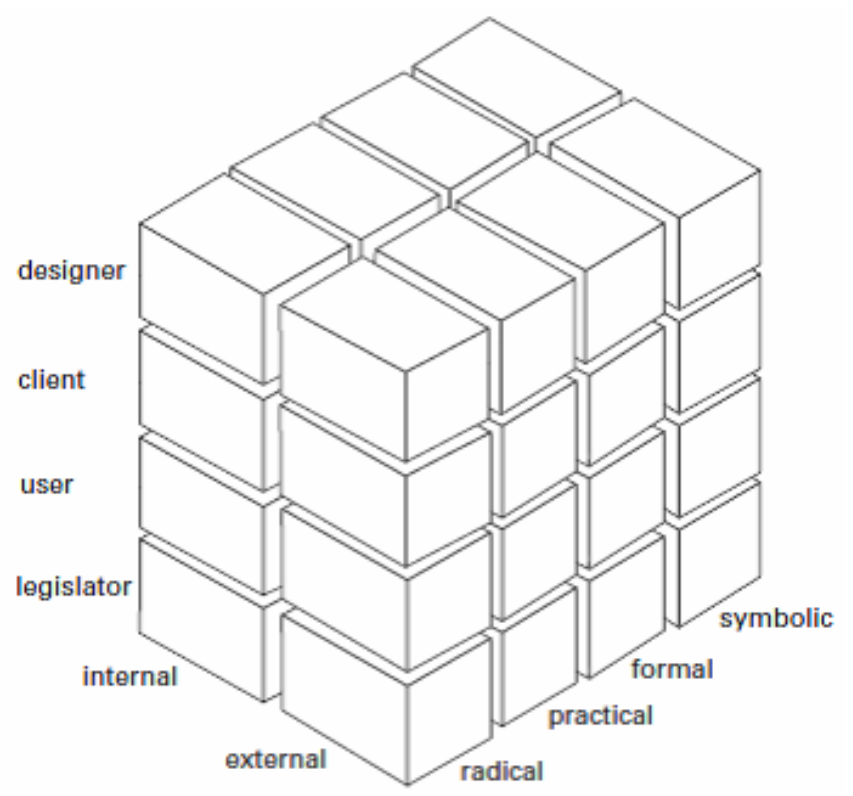

Figure 3 - the model of design problems by Lawson [1, Ch.6, p.107]

Although the assembly process is not part of the current research it can be characterised as follows: the builder resolves construction details and assembles components off-site; structural floor, wall and roof panels are made into a volume and fitted-out by trades in sequence, similar to site construction; site services and concrete works are completed 
before the volumetric modules are transported to site on a flatbed truck so that two, three or four modules may be joined together to form a whole house; and finally, connections, flashings and some smaller components are fixed on site to complete the assembly.

This review of the design process suggests that some improvement in radical constraints, such as client or user inspired space re-arrangements, may be possible but that these would have to be limited by cost and customer service requirements to remain part of the design series. Formal constraint improvements may be limited to user choice of colours and textures which are very subjective by nature and therefore difficult to quantify. Practical constraints offer more potential for speculation and improvement, in particular the technical performance of the modules could benefit from further analysis and evaluation of the initial design criteria and a search for better alternatives. Symbolic constraints offer some prospect for meaningful improvement but only subject to client and designer interaction.

Having better understood the nature of the design process and the design problems as Lawson's model [1], the discussion now turns to the question of which computational design tools are appropriate for making improved design solutions.

\section{COMPUTATIONAL DESIGN TOOLS}

In order to make such a judgement it is useful to briefly review computational design tools capabilities for improving the design solution of the modular homes. The most appropriate tools are then examined in more detail in the sections which follow.

\section{A. Modelling}

For more than thirty years the manufacturing industry has used parametric software to design and model aeroplanes, automobiles and ships. Meanwhile CAD software for construction and architects generally has focused on its representational rather than computational features. This has obscured insight into the logic of design intent, the process whereby the designer can view the different stages of design development or investigate in more detail how changing one variable or parameter might influence the final outcome [5].

Architects who have used parametric modelling have mainly concentrated on geometrical and topological changes to explore how these propagate in real-time and provide new and sometimes unexpected solutions. However parametric modelling has the ability to include other parameters as well. For example material, structural, environmental and human based criteria may be represented as variables and influenced parametrically to enhance understanding of the model's performance in real-time and at real-world scale. However,
Lawson [1] reminds us that these other variables are sources of evaluation to inform synthesis, and not solutions to design problems. Also, as Jabi [6] has noted:

"Very few architects and software developers have taken on the challenge to classify, let alone invent, systems that can accept fundamentally different types of parameters.”

[6, Part 3, p.196]

We might speculate that one of the reasons architects are so reticent to invent such systems is that the tools may be inflexible compared with traditional design techniques. However, and as proposed by this paper, it is also the case that multi-parametric design is central to integrated and interdisciplinary design [7]. Multi-parametric design embraces this integrated process by enabling evaluation and representation of its design process thereby permitting all stakeholders insight into the process itself rather than its outcomes alone [5]. There is clearly a substantial challenge in this task, the complexity of which should not be underestimated. Perhaps one of the key problems with the design process and its solutions is their multivalent nature.

"Design problems are often both multi-dimensional and interactive. Very rarely does any part of a designed thing serve only one purpose.” [1, Ch. 4, p. 56]

The choice of which criteria to focus on and which to ignore becomes the key consideration and generator of potential solutions. Indeed a central criticism of 'parametricism' can be found in its apparent disregard of all but the formal and geometrical parameters. In the process of multi-parametric design criteria may need to be weighted for importance, or they may be a matter of subjective judgement, and then there is also the task of combining them into an overall assessment [1]. In the early stages of design key criteria are analysed, evaluated and synthesised into varied or alternative design solutions whilst benefitting from the adaptability of the parametric model. However a particular problem arises where the architects providing designs for the modular homes may or may not be persuaded to use these techniques.

The current design solutions for the modular homes shown above are considered complete under the traditional design process. Therefore it may be more useful to the client to make use of parametric modelling as a way of capturing the design intent of the architects. A parametric model would then allow further incremental improvements, or even larger variations, to be more easily and integrally generated compared with nonparametric CAD applications. 


\section{B. Simulation}

The testing and evaluation of systems and design models in simulated environments is not something new. As Simon [2] has remarked:

"Simulation, as a technique for achieving understanding and predicting the behaviour of systems, predates of course the digital computer.” [2, Ch.1, p.13]

Simulations are useful in providing new knowledge in two ways. Firstly, the simulated model allows real-scale understanding of behaviour which would be difficult to predict otherwise. Secondly, we can abstract by ignoring or discarding irrelevant criteria, so that it becomes easier to simulate the simplified model's behaviour. Artificial systems and design models have properties that make them, "particularly susceptible to simulation via simplified models.” [2, Ch.1, p.16]

Although we may have analysed the interface between the inner and outer environments and evaluated the simulated solutions we may be no better off in understanding whether the technical performance of the modular homes has been improved as a whole. Simulation of key criteria could provide useful analytical data for evaluative purposes. However, these criteria need to be carefully chosen and their interactions understood before they can be useful for synthesis into alternative design solutions.

"Modern building science techniques have generally only provided methods of predicting how well a design solution will work. They are simply tools of evaluation and give no help at all with synthesis.” [1, Ch.4, p.58]

As the design problem concerns how to improve the modular home's overall performance, the design process is re-defined as the need to understand the behaviour of the whole in relation to its parts in order to provide better synthesised design solutions. A tool for improving each of the modular home's performance criteria in a measured way is needed and this is where optimisation methods are useful, the next computational design tool to be examined.

\section{Optimisation}

“To 'optimise' means to find the best... The process of optimisation describes the synthetic search for this best state within a model, whether of a biological system or architectural or structural system, usually under a set of restrictions, implied or expressed.” [8, Ch.4, p.117]

Simon [2] describes the logic of optimisation methods whereby an 'inner environment' of the design problem is defined by goals or objectives, and the 'outer environment' is represented by parameters or constraints which may or may not be known with certainty. This begins to reveal the complexity of the method required to trade-off interacting performance criteria, and the need for a search process to find this best state in the model. This is called multi-criteria optimisation and may be defined by a Pareto optimisation, a state where one criteria can only improve at the expense of another [8]. Structural economy and reduction in energy consumption are examples of suitable targets for optimisation routines.

Lawson [1] again sounds a cautionary note about using techniques which involve making value judgements between competing criteria in a typically disordered design process as there may be disagreement about the value of alternative optimal design solutions. Nevertheless it seems clear that optimisation could provide useful optimal alternatives, and be a way of linking analysis and simulation more directly to synthesis in design. These algorithmic tools for optimisation techniques will be discussed in more detail.

\section{MULTI-PARAMETRIC DESIGN}

This section explores how parametric design might improve the design process for the modular home series. Although parametric design isn't a new phenomenon, it emerged 50 years ago when Sketchpad was created by Sutherland [9], it has become an area of considerable interest over recent decades. As Sutherland observes:

"It is this ability to store information relating the parts of a drawing to each other that makes Sketchpad most useful."

[9, Ch.1, p.19]

Relating parts to the whole creates an integrated model, but more than that, it is a model whose every past move or action may be queried, hence it is a model in which the design process is made 'explicit', in which an idea is mapped to an intended outcome [10].

Schodek [11] categorises current CAD systems and their parametric capabilities. He defines concept and rendering modellers (Rhino, Autodesk 3DS Max, Sketchup), animation modellers, entity based modellers (2D), component based modellers (Autodesk Revit, Microstation, Archicad) and design development programs (Digital Project, Creo, Solidworks). Each facilitates parametric modelling to varying degrees of complexity, and is useful at different design stages. Design development programs offer the most advanced parametric capabilities (Figure 4), whilst concept and component modellers are more simplified.

The modular home series began as freehand sketches which were then drafted in a component based modeller CAD (Autodesk Revit). However, neither the modeller's simplified parametric capabilities nor its basic functionality were fully 
utilised. Integration of the model is easily improved by linking to related textual data via spreadsheets, whilst remodelling parametrically would increase its value as a record of the architect's design intent. Future changes could then also be more quickly propagated through the model than the current non-parametric model allows.

Because of its integrated nature parametric design is complementary to the analysis and evaluation of other criteria [5] which is termed 'multi-parametric design'. However there are limits to its capabilities and the complexity of related objects within a multi-parametric model can make it 'brittle' or over-constrained so that inflexibility results [12]. Expressing the desire to realise the potential of this emerging interest, Mark Burry has remarked:

“... we have a hunger for far greater computer power to allow the multi-parameter decision-making to take place in realtime.“ [10, Ch.2, p.25]

A design project between SIAL/RMIT, Cox Group and Arup confirmed these findings [13]. It questioned how engineering and architectural expertise, assisted by digital optimization, can promote structural awareness regarding design alterations in the conceptual design stage. An all-encompassing parametric model capable of accommodating any kind of design change to the geometrical setup of a project was found to be inadvisable as it became over-constrained. A design development program (Digital Project), structural analysis package (GSA) and custom scripting achieved these mixed results.

However one benefit of design development programs is their rich data structures which allow the generation of a mesh for finite element analysis (FEA) to simulate structural, thermal, vibration and other behaviours [11]. This direct link between parametric modelling and simulation is based on complex abstractions and embedded material performance criteria. This could be useful if advanced understanding of the structural, material and environmental performance characteristics of the modular homes is required.

A further study by Willis et al [14] describes a multi-criterion parametric housing modeller to generate housing types. Site, roof, massing, solar orientation and type were the criteria considered. The resulting computational framework allowed home builders and customers to generate customised site specific homes. A concept modeller (Rhino), a visual algorithm (Grasshopper) and a scripted discursive shape grammar achieved these results.

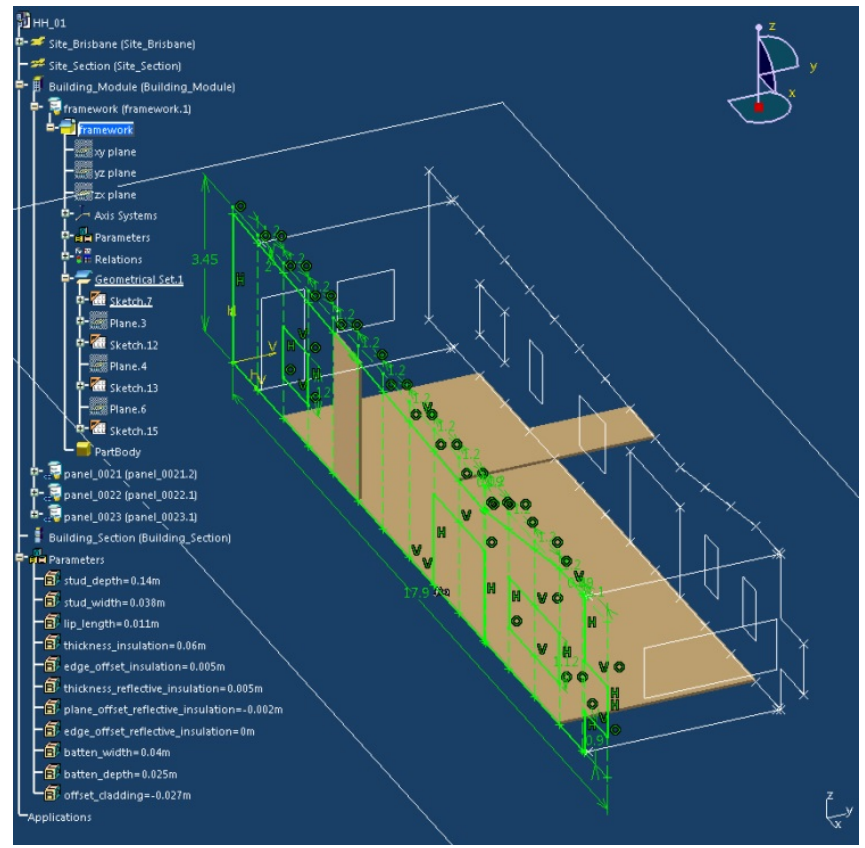

Figure 4 - model of the modular home in Digital Project - the parametric frame, drivers and constraints

This review of multi-parametric design tools suggests that simplified parametric modellers are a better option for improving the current design solution of the modular home series. They are less likely to become over-constrained and promise quicker implementation as they link with widely available add-ons and knowledge bases. Also design constraints including layout, form and style might be more easily captured to represent design intent, or tweaked to provide alternatives for customised options, than advanced parametric modellers allow.

\section{SIMULATION}

It is important to be mindful of the limitations of simulation in the design process and that choosing the right criteria for meaningful analysis does not guarantee integrated solutions. Simulations are just one step on the way, but to be even more useful in the design process feedback from simulations in realtime, or close to it, would assist greatly.

"Scientists have tended to want to develop increasingly precise tools for assessing design... They are purely evaluative tools which do nothing to suggest solutions, but merely assess them after they have been designed.”

[1, Ch.5, p.71]

The limits of computational performance were tested in a study by Burry et al [15] describing a performative approach to the modelling and simulation of built structures focused on integrating physical and digital models. It also aimed for realtime simulation to achieve a materially responsive 
architecture. Tools to achieve this included concept modelers (Rhino), design development programs (Digital Project), visual algorithms and scripted overlays (Grasshopper, C++, Processing and Maya). The results were encouraging:

“... we are not yet able to reach the desired levels of real-time computed feedback on material performance for complex structural arrangements, we are nevertheless getting much closer than we have been in the past.” [15, p.137]

Toth et al [16] successfully developed a cloud-based system which coupled parametric modelling and energy simulation with evaluation in close to real-time, focused on the Joint Contact Centre offices, Brisbane, Australia. For better communication of results these were pushed to a web application displaying design options and performance outcomes side-by-side. Tools used included visual algorithms (Generative Components) and energy analysis software (Energy Plus).

These two examples confirm the importance of the careful choice of criteria for simulation purposes and that computational performance is getting close to being able to incorporate this analysis in real-time. The modular homes series might choose further analysis of the following criteria to improve the design solution: environmental behaviour to determine solar insolation dependent on orientation; energy analysis to establish heating and cooling loads; natural ventilation flows and natural lighting; panel rationalisation or the efficient nesting of panels appropriate to computer numerically controlled (CNC) machining.

\section{MULTI-CRITERIA OPTIMISATION}

Building on the discussion of optimisation above, the goal of multi-criteria optimisation is to improve performance toward some optimal point or points. Improving performance is the process, whilst convergence towards some optimal point is the destination. Rather than convergence to the best a more reasonable goal is to achieve better relative to others, defined by Simon [2] as 'satisficing'.

Multi-criteria optimisation may be achieved using evolutionary optimisation algorithms (EO) which utilise a population based approach. More than one solution contributes to an iteration which then evolves a new population solution after further iteration. The operation stops when one or more termination criteria are met [17]. Goals for optimisation routines which may be appropriate for improvement of the modular home series are many, and may include: design type variations; placement and orientation of buildings; daylight levels and sun hours in relation to views; cost and thermal performance; flows of air and heat; the best use of natural light; and acoustic performance.
An effective study of multi-criteria optimisation using EO’s includes Janssen and Kaushik's [18] exploration of an approach that evolved populations of housing design variants. A template was used to create development and evaluation procedures for a large residential housing project. The EO was executed on a cloud-computing platform.

Evins et al [19] demonstrated multi-criteria optimisation for a residential project in Scotland in their search for higher performance for lower costs in housing designs. They described an optimisation of two objectives - carbon dioxide emissions and cost. The optimisation algorithm used was NSGA-II, and the results were an optimal set of designs illustrated by a Pareto optimal front which graphically illustrated trade-offs from expensive/low carbon dioxide, to cheap/high carbon dioxide levels (Figure 5).

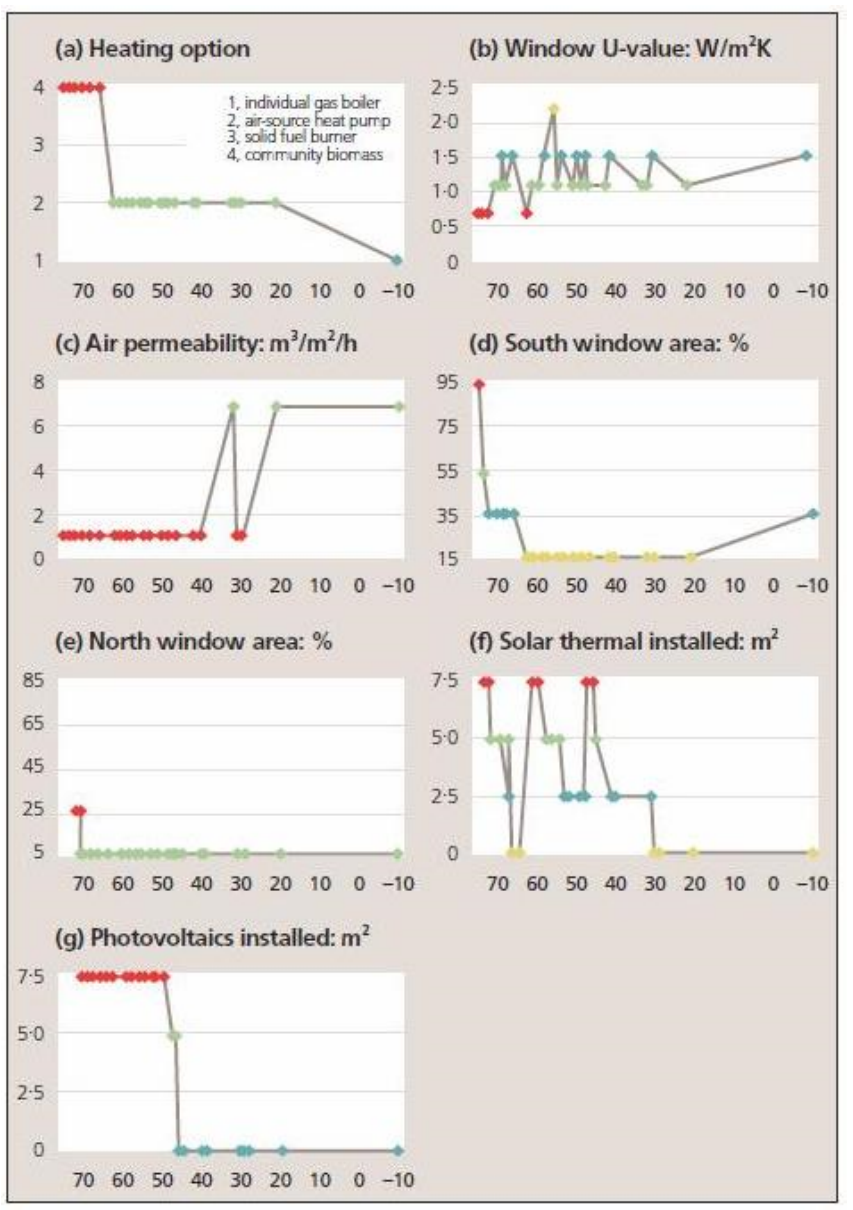

Figure 5 - variations among optimal solutions for a low-carbon dioxide housing problem by Evins et al [19]

They also described the benefits of combining optimisation with parametric design. These included: reduced time per design option trialled (although increased set-up time noted); 
improvements in performance for complex problems; and increased diligence in the design process. The modular home series could capitalise on these benefits as small improvements would result in savings multiplied due to their repeatability.

This brief review has made it clear that simulation is useful when the system is not understood fully or when unpredictability can still occur, whilst optimisation is useful when we understand the design model or system to be improved. As the latter is the case with the modular homes series this method is worth exploring further, focusing on some of the criteria already noted above.

\section{CONCLUSION}

This paper has described computational design tools which can improve design solutions for a modular homes series. The design process for achieving these improvements has been based on Lawson's [1] model, whilst his reservations regarding computational design's ability to contribute have been noted:

“... getting a computer to fully, automatically and accurately understand all the modes of thought about a building that an architect uses is probably not a realisable goal.” [20, Ch.6, p.79]

By confirming that computational design tools are available to enhance the outcomes of the design process, this research has also addressed one of the key characteristics and concerns of prefabricated and modular off-site assembly which is that it requires more up-front design work than on-site construction methodologies. Therefore application of these tools will enhance integration of this inter-disciplinary design process leading to better performing design solutions for the modular homes series. This should also translate into a quicker and more streamlined design and build phase, and may result in reduced cost and waste in assembly and operation.

Some of the tools which achieve these improvements require a different way of thinking to the traditional design process. Lawson [1] concludes that design problems require divergent thinking, but concedes that at times the design process involves convergent thinking when a logical process might lead us to one or a number of optimal solutions.

"The ability to communicate across fields - the common ground - comes from the fact that all who use computers in complex ways are using computers to design, or to participate in the process of design.” [2, Ch.3, p.83]

Parametric modelling facilitates this logical process, and therefore assists with discussions across fields. It is also argued that being able to evaluate design problems in an integrated way using multi-criteria optimisation methods would further improve this process in addition to offering greater performance improvements to the design solutions.

Having defined the design problem and explored applicable computational design methods several questions are raised for future research, including: the value of multi-parametric design using concept and component based modellers; the establishment of key criteria for simulation; and, because of our understanding of the design model to be improved, optimization searches using EO's to define Pareto optimal design solutions provides the focus for this further research. 


\section{ACKNOWLEDGMENT}

Thankyou to Dr Sean Hanna, Bartlett School of Architecture, University College London, UK for guidance regarding multi-criteria optimisation.

\section{REFERENCES}

[1] B. Lawson, How Designers Think - The Design Process Demystified, 4th ed. UK: Architectural Press, 2005.

[2] H. A. Simon, The Sciences of the Artificial. Cambridge, Massachusetts: MIT Press, 1969.

[3] M. McGill, "A Visual Approach for Exploring Computational Design," MSc thesis, Department of Architecture, MIT, Cambridge, MA, USA, 2001

[4] C. Alexander, The Timeless Way of Building. New York: Oxford University Press, 1979

[5] K. Terzidis, Algorithmic Architecture. Oxford, UK: Elsevier Ltd., 2006.

[6] W. Jabi, Parametric Design for Architecture. London, UK: Laurence King Publishing Ltd., 2013.

[7] A. Menges and S. Ahlquist, Computational Design Thinking. UK: John Wiley \& Sons Ltd., 2011.

[8] J. Burry and M. Burry, "Optimisation," in The New Mathematics of Architecture, ed London: Thames and Hudson Ltd., 2010.

[9] I. E. Sutherland, "Sketchpad: a man-machine graphical communication system," PhD dissertation, Massachusetts Institute of Technology, 1963.

[10] M. Burry, Scripting Cultures: Architectural Design and Programming. UK: John Wiley \& Sons Ltd., 2011.
[11] D. Schodek, M. Bechthold, K. Griggs, K. M. Kao, and M. Steinberg, Digital Design and Manufacturing: CAD/CAM Applications in Architecture and Design. Hoboken, New Jersey: John Wiley \& Sons Inc., 2005.

[12] D. Davis, "Modelled on Software Engineering: Flexible Parametric Models in the Practice of Architecture," PhD dissertation, School of Architecture and Design, RMIT University, Melbourne, VIC, Australia, 2013.

[13] D. Holzer, R. Hough, and M. Burry, "Parametric design and structural optimisation for early design exploration," International Journal of Architectural Computing, vol. 05, 2008.

[14] B. Willis, T. Hemsath, and S. Hardy, "A parametric multi-criterion housing typology," presented at the ACADIA12, San Fransisco, CA, USA, 2012.

[15] J. Burry, M. Burry, M. Tamke, M. R. Thomsen, P. Ayres, and e. al, "Process through practice: synthesizing a novel design and production ecology through Dermoid," presented at the ACADIA12, San Francisco, CA, USA, 2012.

[16] B. Toth, F. Salim, R. Drogemuller, J. H. Frazer, and J. Burry, "Closing the loop of design and analysis : Parametric modelling tools for early decision support," presented at the CAADRIA11, Newcastle, NSW, Australia, 2011.

[17] K. Deb, "Multi-objective optimisation using evolutionary algorithms: an introduction," in Multi-objective evolutionary optimisation for product design and manufacturing, L. Wang, A. H. C. Ng, and K. Deb, Eds., ed London: Springer, 2011, pp. 3-34.

[18] P. Janssen and V. Kaushik, "Evolutionary design of housing: a template for deveopment and evaluation procedures," presented at the Cutting Edge: 47th International Conference of ANZAScA, Sydney, NSW, Australia, 2013.

[19] R. Evins, S. C. Joyce, P. Pointer, S. Sharma, R. Valdyanathan, and C. Williams, "Multi-objective design optimisation: getting more for less," Civil Engineering Special Issue 165, vol. 165, pp. 5-10, 2012. 